\title{
GENERATION OF THE SEED MAGNETIC FIELD
}

\author{
A. LAZARIAN \\ DAMTP, University of Cambridge, UK
}

\begin{abstract}
Two mechanisms of the galactic seed field generation are discussed. One of the mechanisms implies a direct generation of the seed magnetic field through a battery process (Lazarian 1992a). The other accounts for the possibility of the preliminary amplification of the magnetic field on a scale of molecular clouds (Lazarian 1992b). This means that the galactic dynamo can feed on the non-zero average value of the magnetic field amplified by such a small-scale dynamo. It is shown that both mechanisms can generate an adequate seed field. These two scenarios of the seed magnetic field generation can be distinguished by an analysis of the present day galactic magnetic structure.
\end{abstract}

Key words: seed field - dynamo - molecular clouds - ISM

\section{Introduction}

Cosmic magnetic fields are an essential part of the interstellar matter. They influence diffusion processes, formation of stars and other astrophysical processes.

A very weak initial field can be amplified considerably by a dynamo action on the galactic scale (Moffatt 1978, Parker 1979, Krause et al. 1980), but for the dynamo to take place some weak field must already exist.

Several mechanisms have been proposed for solving the problem (Catani et al. 1966, Lesch et.al. 1989). Unfortunately, they cannot generate a sufficiently large seed magnetic field without challenging our current understanding of the early stages of galactic evolution (Rees 1987). The $\alpha-w$ dynamo exponentially amplifies the initial magnetic field $B_{0}$ to its present value $B\left(\sim 10^{-6} \mathrm{G}\right)$ in a time $t$ i.e. $B=B_{\circ} \exp (t / \tau)$, where $\tau$ is a characteristic growth time, which should be at least greater than the typical rotation period of a galaxy $\sim 3 \times 10^{8}$ years. This gives a lower limit on the initial magnetic field: $B_{0}>10^{-21} \mathrm{G}$.

It has been shown (Lazarian 1992a) that in the ISM there exist effective battery mechanisms for generation of the seed magnetic field. These mechanisms can be considered to be of the Biermann type, because they appear wherever electronic temperature and density gradients are not parallel, i.e. similar to the condition required for the original Biermann mechanism to operate (Biermann 1950). Thermal energy is transformed into electrical energy, and thus the e.m.f. can be called thermally generated. However, in this paper, it will be referred to as diffusion e.m.f. so as to emphasise the role of diffusion processes in its generation. These mechanisms are effective enough to seed the ISM with magnetic fields. Then these magnetic fields will be amplified by a turbulent dynamo in a hot phase of the ISM (Kulsrud et.al.) and by a dynamo in molecular clouds (Lazarian 1992b) to a level which will influence star formation processes and further evolution of the first generation of stars.

Two possible scenarios of the galactic seed field generation are discussed here. According to the first scenario, the entire seed field was generated by the battery (Lazarian 1992a). Previous attempts to account for sufficient seed magnetic field 
generation have been unsuccessful as a result of the implicit assumption of the uniformity of the ISM.

The idea for the second possibility is similar to that of Rees (1987). He discussed a magnetic field ejected from stars ${ }^{1}$ and pointed out that "the resultant large-scale flux would grow ... as the square root of the number of stars", but "predominant present-day field could have acquired a large-scale structure provided that only the large-scale component underwent dynamo amplification". In contrast, it is shown in the present paper that the regular magnetic field can emerge from the non-zero average of the random magnetic loops which are generated on the scale of molecular clouds, prior to the time of active star formation (Lazarian 1992b). This is a crucial difference, as, according to Rees (1987), the magnetic field problem has two aspects;

1. how to account for the generation of the sufficient seed magnetic field.

2. what were the physical processes (e.g. star formation) like at the early stages of galactic evolution.

If the seed field were to appear in the ISM after the first generation of stars had evolved, then the formation of early stars would be quite different from the present-day one. In contrast, here, we advocate the idea, that the magnetic field is rapidly generated at the scales which are large in comparison with the ones of star formation sites, but small in comparison with a size of a galaxy. The idea of magnetic field amplification in molecular clouds was put forward by BisnovatyiKogan et al. (1976). In the present paper, it is combined with the idea of Rees that contributions of random small-scale magnetic loops can add up in a large-scale flux, which in turn can serve as a galactic seed field. Therefore, in this scenario both aspects of the seed magnetic field problem find their solution.

One can choose between the two scenarios by analyzing present-day magnetic structure.

\section{Battery Generated Seed Field}

Consider a generalised Ohm's law (Alfvén et al. 1963): $\mathbf{E}(x)=-\frac{1}{n_{\mathrm{e}}(\mathbf{x}) \mathrm{e}} \nabla p_{\mathrm{e}}(\mathbf{x})$, where $n_{\mathrm{e}}(\mathbf{x})$ is the density of electrons, e is the electron charge, $p_{\mathrm{e}}(\mathbf{x})$ is the electronic pressure. To generate an e.m.f. a non-zero curl $E$ is needed:

$$
\mathcal{E}=\nabla \times \mathbf{E}=-\frac{1}{\mathrm{e}} \nabla \times\left(\frac{\nabla p_{\mathrm{e}}(\mathbf{x})}{n_{\mathrm{e}}(\mathbf{x})}\right)=-\frac{1}{\mathrm{e} n_{\mathrm{e}}(\mathbf{x})} \nabla T_{\mathrm{e}}(\mathbf{x}) \times \nabla n_{\mathrm{e}}(\mathbf{x})
$$

Consider a partially ionised gas consisting of electrons, ions and atoms (molecules) with concentrations $n_{\mathrm{e}}(\mathbf{x}), n_{\mathrm{i}}(\mathbf{x}), n_{\mathrm{a}}(\mathbf{x})$ respectively, sutisfying

$$
k T(\mathbf{x}) n_{\mathrm{e}}(\mathbf{x})+k T(\mathbf{x}) n_{\mathrm{i}}(\mathbf{x})+k T(\mathbf{x}) n_{\mathrm{a}}(\mathbf{x})=p_{\circ}=\text { const }
$$

where temperatures of all the components are equal. It is easy to see, that electronic pressure $p_{\mathrm{e}}(\mathbf{x})$ can vary while $p_{0}=$ const. The degree of charge separation given by $\frac{1}{4 \pi} \operatorname{div} E$ is so small that $n_{\mathrm{e}}(\mathbf{x})$ is very nearly $n_{\mathrm{i}}(\mathbf{x})$. Thus, it is possible to express

${ }^{1}$ In stars the magnetic fields could originate due to the existence of the Beiramnn mechanism (Biermann 1950, Mestel et al. 1983). 
the temperature from the previous expression: $T_{\mathrm{e}}(\mathbf{x})=\frac{p_{0}}{k\left(2 n_{\mathrm{e}}(\mathbf{X})+n_{\mathrm{e}}(\mathbf{x})\right)}$. According to Eq. (1),

$$
\nabla \times \mathbf{E}=\frac{4 p_{\mathrm{o}}}{\pi e n_{\mathrm{e}}(\mathbf{x})\left(2 n_{\mathrm{e}}(\mathbf{x})+n_{\mathrm{a}}(\mathbf{x})\right)^{2}} \nabla n_{\mathrm{a}}(\mathbf{x}) \times \nabla n_{\mathrm{e}}(\mathbf{x})
$$

In the case of a small charged impurity in neutral gas, $\nabla \times \mathbf{E} \approx \frac{4 p_{0}}{\pi e n_{\mathrm{e}}(\mathbf{x}) n_{\mathrm{a}}^{2}(\mathbf{X})} \nabla n_{\mathrm{a}}(\mathbf{x}) \times$ $\nabla n_{\mathrm{e}}(\mathbf{x})$.

It is well known that the concentration of ions and electrons is different in the hot, warm and cold phases of the ISM (see Shull 1987). Even allowing for different sources of the atom and electron gradients these should not generally coincide. A relatively small impurity of atoms in plasma, and vice versa, can result in the appearance of an e.m.f. ${ }^{2} \mathrm{~A}$ more accurate treatment of the problem is given in Lazarian (1992a), where it is shown that the conditions for e.m.f. generation emerge on the border area of the molecular cloud-plasma if the temperature gradient is applied to plasma. The magnetic field increment $\triangle B$ through the surface $S$ over the time $\Delta t$ can be estimated as $\Delta B \sim \frac{c \mathcal{E}}{S} \Delta t$. For a galaxy, $S \sim H \times R$, where $H$ is a half thickness of the atomic layer $\sim 200 \mathrm{pc}, R$ is the galactic radii $\sim 10^{4} \mathrm{pc}$. If the temperature gradient existing in the hot media between the galactic centre and its edge is $\sim 10^{6} \mathrm{~K}$, then $\triangle B \sim 10^{-17} \mathrm{G}$.

The field consists of closed circular loops in the galactic plane, but the rise of warm gas elements and the inflation of magnetic field lines by cosmic rays are essential for the $\alpha-w$ dynamo on the galactic scale and result in the formation of dipole and further quadrupole configurations through the twisting of the magnetic field lines and a reconnection process. A magnetic field of quadrupole configuration can be amplified by the $\alpha-\omega$ dynamo (Parker 1979).

According to Rees (1987), even a field of $10^{-18} \mathrm{G}$ could suffice as a seed field on a galactic scale. Thus, a diffusion generated magnetic field can serve as a seed galactic field. Note that this seed field has a regular structure.

\section{Seed Field of Statistical Nature}

The process of magnetic field generation by a diffusion e.m.f. is more efficient on smaller scales. A similar statement is valid for the dynamo processes and it is natural to assume that the state of equipartition is reached more quickly at these scales. Rigorous calculations of the magnetic noise spectrum and its backreaction on the turbulence (Kulsrud et al. 1992) prove these arguments. However, as the turbulence in the ISM is a very complex problem (Lazarian 1992c,d), we will avoid a general treatment of the problem and consider only magnetic fields associated with molecular clouds (Lazarian 1992b).

The magnetic fields generated both inside and outside molecular clouds (Lazarian 1992b) can be expanded over the eigenfunctions $\mathbf{C}_{n}$ of the problem of magnetic field excitation (Ruzmaikin et al. 1988). Indeed, random magnetic loops can be approximated by derivatives of delta functions $\mathbf{h}_{i} l_{i} \delta^{\prime}\left(\mathbf{r}-\mathbf{r}_{i}\right)$, where $h_{i}$ and $l_{i}$ are

2 The process of e.m.f. generation discussed is similar to the process of e.m.f. generation in a thermacouple where two different metals in contact are subjected to a temperature gradient. In our case, different phases of the ISM serve as different metals. 
the amplitude and scale of the loop at the position $\mathbf{r}_{i}$. Therefore (Ruzmaikin et al. 1988),

$$
K_{n} \sim \frac{1}{3} \frac{1}{\int C_{n}^{2} \mathrm{~d}^{3} \mathbf{r}} \sum_{i=1}^{N} \int \mathbf{C}_{n}(\mathbf{r}) \cdot \mathbf{h}_{i} l_{i} \delta^{\prime}\left(\mathbf{r}-\mathbf{r}_{i}\right) \mathrm{d}^{3} \mathbf{r}
$$

where the coefficient $\frac{1}{3}$ accounts for the random orientation of the magnetic loops and $N$ is the number of magnetic loops in the volume over which the integration is performed. As the coefficients $K_{n}$ are random variables themselves, we may find their dispersions. For example, for the lowest eigenfunction, $\mathbf{C}_{1}=C \mathbf{i}_{\varphi}$ and $\sqrt{\left\langle K_{1}^{2}\right\rangle} \sim \frac{1}{3} \cdot \frac{\sqrt{\left\langle h^{2}\right\rangle}}{C \sqrt{N}} \frac{\sqrt{\left\langle 1^{2}\right\rangle}}{L}$, where $\mathbf{i}_{\varphi}$ is a unit azimuthal vector, $L$ is a width of the mode localisation and angular brackets $\langle\cdots\rangle$ denote the averaging procedure. Thus the seed magnetic field for $n=1$ mode can be estimated as $B_{\varphi 1} \sim \frac{1}{3} \frac{\sqrt{\left\langle h^{2}\right\rangle}}{\sqrt{N}} \frac{\sqrt{\left\langle l^{2}\right\rangle}}{L}$. The number of cells can be estimated as $N \sim \frac{V_{g}}{\left\langle l^{2}\right\rangle^{3 / 2}}$, where $V_{g}$ is a galactic volume $\sim R^{2} H$ (and thus $N \sim 10^{6}$ ). Assuming $\sqrt{\left\langle h^{2}\right\rangle} \sim 10^{-6} \mathrm{G}, \sqrt{\left\langle l^{2}\right\rangle} \sim 10 \mathrm{pc}$ and $L \sim R \simeq 10^{4} \mathrm{pc}$, one obtains $B_{\varphi 1} \sim \frac{10^{-6}}{10^{3}} \times \frac{1}{10^{3}} \mathrm{G} \sim 10^{-12} \mathrm{G}$. This estimate is greater than that for the large scale magnetic field generated by the diffusion e.m.f. However, the efficiency of the magnetic field generation strongly depends on the characteristic size of the random magnetic loops $B_{\varphi} \sim \sqrt{\left\langle h^{2}\right\rangle} \times \frac{\left\langle l^{2}\right\rangle^{5 / 4}}{R^{2} \sqrt{H}}$. If we adopt estimates for the size of magnetic loops from Kulsrud et al. (1992), we come to a seed field estimate of less then $10^{-17} \mathrm{G}$. This means that at although the small scales, the. magnetic field is at equipartition and star formation is similar to a present-day one (the second aspect of the seed field problem), the battery (see section 2) is responsible for the generation of galactic seed field.

\section{Discussion}

Two possible scenarios of the seed magnetic field generation at the earliest stages of galactic evolution have been discussed. Both of them can account for the generation of a sufficient magnetic field. Which of the mechanisms is more efficient depends on the detail processes in the ISM. If the generated random magnetic field has a relatively large characteristic scale, a statistical seed field is favourable. However, little is known about early stages of galactic evolution, and one can not rule out that a large-scale magnetic field generated by a battery can serve as a galactic seed field. The two possibilities can be distinguished by analyzing the configuration of the present-day galactic magnetic field. For a random seed magnetic field one can expect to observe reversals of the present-day field direction along the galactic radius (Ruzmaikin et al. 1988), while the field amplified from the regular large-scale battery-generated seed field should be more uniform.

As for the second aspect of the seed field problem (Rees 1987) (i.e. the dynamics of astrophysical processes influenced by magnetic field at the early stages of galactic evolution), some of the processes should not be different from those of the presentday. For example, the magnetic field quickly $\left(\sim 10^{7}\right.$ years) reaches equipartition at the small scales (as compared with the galactic size). However these scales are 
large in comparison with the scale of star forming site, as far as star formation is concerned. For other processes (Rees 1987), the situation is less clear. For example, there are differences of the magnetic type for the early supernova, as in this case the expansion of the explosion shell took place in a medium with just random magnetic field.

Further evolution of the seed field depends on the details of the galactic dynamo. A very important question concerns the nature and the evolution of the galactic the alpha-effect. Present day theories (see Ruzmaikin et.al. 1988) assume that alpha-effect is independent of galactic evolution. This seems to be a risky assumption at least at the earliest stages of evolution. Furthermore, the characteristics of turbulence in galaxies are poorly known (see Lazarian 1992d), while attempts of attributing alpha-effect to supernova explosions result in rather discouraging estimates (see Ferrière 1992). An alternative physical process which may produce the alpha-effect is the Parker instability. However this process is a nonlinear one and thus the galactic dynamo may need a different mathematical treatment.

\section{Acknowledgements}

The author is greatly indebted to L. Mestel, K. Moffatt, and N. Weiss for their encouragement. I take great pleasure in expressing my gratitude to M. Rees, a discussion with whom initiated this work. This research is supported by an Isaac Newton Scholarship. Finantial assistance of the LOC is acknowledged.

\section{References}

Alfvén, H. and Falthammar, C.-G.: 1963, Cosmical Electrodynamics, Oxford, Clarendon Press, p.109.

Biermann, L. : 1950, Z. Naturf. A., 5, 65.

Bisnovatyi-Kogan, G.S. \& Ruzmaikin, A.A.: 1976, Astrophys. Space Sci., 42, 401.

Catani, D. and Sacchi, C.: 1966, Nuovo Cimento, XLVIB, No. 2, 258.

Ferrière, K.Mf.: 1992, $A p J, 389,286$.

Krause, F., Rädler, K.-H.: 1980, Mean-Field Magnetohydrodynamics and Dynamo Theory, Akademie-Verlag, Berlin, p.

Kulsrud, R.M. and Anderson, S.W.: 1992, A p.J., 396, 606.

Lazarian A.: 1992a, $A \mathcal{G} A, 264,32$.

Lazarian A.: 1992b, Magnetic field generation within molecular clouds in The Cosmic Dynamo, ed. F. Krause, K.H. Rädler and G. Rüdiger, Kluwer: Potsdam, p.

Lazarian A.: 1992c, Statistical method for astrophsical turbulence invesgation in The Cosmic Dynamo, ed. F. Krause, K.H. Rädler and G. Rüdiger, Kluwer: Potsdam, p.

Lazarian A.: 1992d, Astron. and Astrophys. Transactions, 3, 33.

Lesch, H., Crusius, A., Schlickeiser, R. and Wielebinski, R.: 1989, $A \& A, 217,99$.

Mestel, L. and Moss, D.L.: 1983, MNRAS, 204, 557.

Moffatt, H.K.: 1978, Magnetic Field Generation in Electric Fluids, Cambridge University Press.

Parker, E.N.: 1979, Cosmical Magnetic Fields, Clarendon Press: Oxford.

Rees, M.J.: 1987, QJRAS, 28, 197.

Ruzmaikin, A. A., Sokolov D. D. and Shukurov A. M.: 1988, Magnetic fields of Galaxies. Kluwer Acad. Publ.

Shull, J.M.: 1987, Interstellar Processes, eds. Hollenbach, D.J. and Thronson, H.A., Reidel: Dordrecht, p.225. 